\title{
The Fire Alarm System based on Electric Power Carrier in the Research and Design
}

\author{
Wu lei \\ Wuhan Institute of Shipbuilding Technology \\ 89370742@qq.com \\ Wuhan China
}

\begin{abstract}
With the development of social economy and the progress of science and technology, the dangers of fire for human is obvious. Therefore, carries on the effective prevention and control, reduce the loss brought by the fire to the smallest degree is the key of the current research on fire prevention and control technology, develop all kinds of fire alarm device for this people, common are based on the cable network and wireless network
\end{abstract}

Fire alarm system, but these alarm system construction cost is higher, difficult to maintain, poor reliability, are not suitable for use in the bad environment, so a single based on cable or wireless fire alarm system can not be widely used. This paper will design a set of independent type fire alarm system based on electric power carrier. This system adopts the power carrier communication technology, electric power carrier as a unique communication mode in the power system, with the existing power line as a carrier to transmit the fire alarm signal, the biggest characteristic is don't need to

Set up network and daily maintenance, so long as has the wires, can for data transfer, is not affected by cable network wiring and wireless network signal strength, the influence of can realize a wide range of coverage. Therefore, independent type fire alarm system based on electric power carrier wave and single compared based on wired and wireless fire alarm system, not only the function is all ready, low cost, flexible organization and system.

Keywords- power line communication;Lwip protocol;C/S and $\mathrm{B} / \mathrm{S}$ hybrid architecture; detache; the fire alarm

\section{INTRODUCTION}

Fire is a kind of harm great disasters, not only can consume large quantities of wealth immediately and even harm to people's lives. Especially modernization since the increase of the high-rise building, the layout of the building and the materials made of fire rescue and fire fighting harder difficulty, if the fire before or occurs can be found in time, and take the corresponding early warning or fire extinguishing measures, then the fire damage will be reduced to a minimum, therefore, automatic fire alarm system plays a more and more important role. Along with the development of the modernization and economy, fire alarm system related equipment has been rapid development. Research shows that in the city of high-rise buildings, shopping malls, hotels, large library, universities and other public occasions, automatic fire alarm system play an important role. In the automatic fire alarm system in the application of all kinds of occasions, the sensors need to quickly determine the characteristics of the fire early signals, such as temperature, smoke, combustible gas, thus making predictions to fire and alarm. Early fire, for example, because of the burning material decomposition, a large amount of carbon monoxide and other toxic gas evolution, people may be in the unconscious of carbon monoxide poisoning, unable to escape so that endanger the safety of life. So the smoke sensor as monitoring of automatic fire alarm system, will judge the alarm at the early stages of the fuel combustion, can to a certain extent, reduce the loss from the fire. At the same time, according to the different options of applications reasonable mode of transmission, automatic fire alarm system was applied to urban high-rise buildings, smart home, even in remote mountainous area. But in some intelligent degree is not high, the same wholesale markets and market, due to technical problems, such as fire detection accuracy or display problems such as funds, management problems, there is still a major fire hazards. Therefore, design a set of high reliability, low cost and wide applications of automatic fire alarm system, is an imminent problem. Electric Power carrier Communication, that is, the Power Line Carrie Communication, is refers to the use of existing Power Line as the media of Communication technology to transmit signal. It is neither in the traditional sense of the wired communication, also overcomes the disadvantages of the wireless communication. Need to wiring, building blocks, as long as there is power, can for information transmission, it is easier to implement than cable communications wiring, is relatively close range wireless communication technology such as ZigBee is easier to achieve network, so more and more widely used. In the past decade, the power system of power carrier communication technology is used only for transfer rate is low, such as remote meter reading, etc. In recent years with the development of intelligent building and the diversification of communication and transport, electric power carrier communication technology to a new kind of communication transmission technology, to provide customers with the services required, such as component family LAN in order to realize the \"four network union\", end users connected to the power line will get to the Internet, in the true sense of home automation. However, at present our country special communication network coverage is still small, but power line laying length to the second in the world, if we can make full use of the advantage of the power line communication, to provide users with Internet services, will be on the reform of telecommunications and the Internet bring innovative. 


\section{SUMMARY OF FIRE ALARM SYSTEM BASED ON ELECTRIC POWER CARRIER}

Fire alarm system is in an important part in the fire command system, in the whole fire control management system has a very important role. Good and effective fire alarm system can be released in time for the fire alarm information, and to evacuate, ensure people's lives and property losses. Common fire alarm system are based on the cable network and wireless network alarm system, but the system has the high construction cost, difficult to maintain, or poor reliability, are not suitable for use in harsh conditions such as faults, so can't have a wide range of applications.

In order to solve the limitations of wired and wireless system, this paper designed a kind of independent type fire alarm system based on electric power carrier. The system is the biggest characteristic is to use electric power carrier wave technology to break the limitations of the traditional fire monitoring system. The system is divided into four parts: 1 . The fire detection device; 2 . Data communication module; 3. Monitor the gateway; 4. The data receiving terminal. Fire detection devices including distribution in each probe point smoke sensor, is mainly responsible for collecting information and control the smoke concentration. Each sensor is connected to the microcontroller, in this system, sensor, the smoke concentration of collected information by single chip microcomputer to power carrier module, power by $220 \mathrm{v}$ power line carrier module, the data is transmitted to the receiver power carrier module, the receiver module power carrier to transmit the data to monitor gateway, monitor gateway is mainly composed of single-chip computer and the router. Composition through a router connected to the data transmitted to the monitoring in the form of sets of interface communication mobile terminal, and the data receiving terminal is a wireless laptops, open the upper machine, through a set of interfaces to connect server, PC can get data from the server, and remote monitoring.

\section{THE RESEARCH CONTENT AND ORGANIZATIONAL STRUCTURE}

Smoke is one of the characteristics of when the fire broke out the over view, the desire to achieve the first part is the function of the fire detection system, on the quantity of smoke was collected. The second part in the system, alarm signal transmission network, the need to choose the appropriate transfer protocol and use the transmission characteristics of this system. With the development of network communication technology, through the power line to transmit data gradually become a reality, half duplex USES FSK modulation power carrier chip, it can be collected smoke concentration information transmission to the power line. So this article emphatically discusses the basic concept and implementation process of the electric power carrier wave communication, and introduces the st7540 based on FSK power carrier chips. In monitoring the gateway module, the system choose ARM chips and family common routers, and through the Lwip protocol and the technology of dynamic DNS. In the third part of the system, the alarm signal at the receiving end, in order to realize the alarm data receiving and display, so in this paper, the client/server $(\mathrm{C} / \mathrm{S})$ architecture and socket communication process was introduced in brief. Because the c \# computer language has simplicity, cross-platform and portability, the article chose the $\mathrm{c} \#$ programming language data receiving process.

This topic mainly studies the following tasks:

(1) On dependent type of fire smoke alarm system structure analysis and the overall scheme design. Through discussing the advantages and disadvantages of the traditional fire alarm system and the characteristics of power carrier technology, to solve how to meet the requirements of the performance of the system using power line communication, and puts forward the scheme design.

(2) The design of the data communication module. In the comparison of the traditional communication mode and the advantages and disadvantages of power line communication mode, choose mainly power carrier transport. And through discussing the basic principle and working process of the electric power carrier communication technology, puts forward adopting half duplex synchronous/asynchronous FSK modem - HL PLC chip.

(3) To monitor the design of the gateway. Monitor gateway is composed of ARM and routers, including domain dynamic analysis to dynamic IP, so that the system through the TCP/IP protocol implementation surveillance gateway and the client Socket communication. This part is mainly completed by ordinary home routers, domain dynamic analysis will be on a specific port and forward the information to set the IP network.

(4) PC interface design. This paper expounds the construction of the PC interface environment and socket communication principle, discussed the client browser and the web server implementation process, and discusses how to through the PC interface to realize the monitoring of smoke sensor.

\section{FIRE ALARM SYSTEM DESIGN SCHEME}

\section{A. Total design scheme of fire alarm system design}

The fire alarm system based on electric power carrier is divided into four parts: the first part for fire detection device. The second part is the data communication module. The fourth part is the data receiving terminal. Fire detection devices including distribution in each probe point smoke sensor, is mainly responsible for collecting information and control the smoke concentration. Each sensor is connected to the microcontroller, in this system, the smoke sensor will undertake the smoke concentration of collected information by single chip microcomputer to power carrier module. Data communication module includes power carrier module of sending and receiving data, sending the power carrier module receives the information, the smoke concentration of data transmission over a $220 \mathrm{v}$ power line power carrier module at the receiving end, at the receiving end, a second power carrier module to transfer the data to monitor the ARM of the gateway. Monitor gateway is mainly composed of ARM and ordinary home routers.

Through connected to the router will smoke concentration data transmitted to the monitoring terminal in the form of sets of interface communication, and the data receiving terminal is a wireless laptops, open the upper machine, through a set of interfaces to connect the 
server, can receive smoke concentration data from the SCM server, the client browser to the remote monitoring. Receiving terminal besides can use wireless lap tops, still can use other mobile devices.

The real-time information sent to the mobile terminal. To realize the user through the domain name to access the query, single-chip should be loaded in the MCU TCP/IP protocol stack (LwIP) and HTTP server program. The initialization process of single-chip microcomputer and LwIP protocol stack in the below mentioned. The router is responsible for dynamic IP domain url is mapped to a family. Router this function by \"peanuts।", |"peanuts $\backslash "$ configuration method of workflow and the router will be detailed in chapter 4.

\section{B. The design on monitoring system of data collection}

Sensor as a data collection terminal, is one of the core components of smoke monitoring module. Therefore, sensor selection is essential to the sensitivity and reliability of the system. Most of the happening of the fire with the release of the burning of combustible materials and flammable gas, so smoke and combustible gas is an important characteristic in front of the fire. Therefore, we need to choose a suitable sensor type, can not only detect the combustible gas in the air, by the ability to recognize a fire smoke concentration, and thus for the fire forecasting and prevention. According to the requirements of fire alarm system design, and through consulting a large number of sensors related literature, the system adopts MQ - 2 smoke sensor as the data collection. When the air temperature of 200 300 range, silica adsorption oxygen in the air, causing less electron density in the semiconductor of resistance increase. In contact with smoke sensor resistance is produced on the surface of the change. Namely, MQ - 2 smoke sensor, combustible gas in the air, such as liquefied petroleum gas, hydrogen, alcohol, methane, smoke, etc.) the concentration of the converted into analog signals, analog signal by Analog to Digital Converter circuit of sensor is converted into digital signal and then sent to the single-chip microcomputer, completed by single chip microcomputer data processing, alarm control, sensor identification, etc.

Data acquisition terminal in this system is mainly composed of multiple smoke sensor. Multiple smoke fumes smoke sensors to collect more data, and connect the same piece of MCU, MCU through RXD, TXD connection module, the electric power carrier and transmit data in the form of serial port, serial port baud rate to 9600. Smoke sensor for two-way signal output, when the output of TTL signals for low power signal lights at ordinary times.

\section{Power carrier communication module design}

Power carrier communication terminal module as the fire alarm system, is an important part of data transmission, therefore, the electric power carrier wave chip selection is crucial, this system choose HLPLCS520F chip as power carrier module of chip. HLPLCS520F inside integrated digital signal processor chip and modem, in addition also integrates the $\mathrm{I} / \mathrm{O}$ module, $\mathrm{A} / \mathrm{D}$ module, general asynchronous transceiver transmitter, etc. Chips working temperature to $40{ }^{\circ} \mathrm{C}$ to $85{ }^{\circ} \mathrm{C}$, the working voltage of 3.3 $\mathrm{V}$ to $5.5 \mathrm{~V}$. Carrier center frequency of $110 \mathrm{KHZ}$, chip serial port baud rate to $1200 \mathrm{-bit} / \mathrm{s}$, and $2400 \mathrm{-bit} / \mathrm{s}$, and $4800 \mathrm{bit} / \mathrm{s}$, and 9600 - bit/s, HLPLCS520F chip need to provide $6.5 \mathrm{v}-20 \mathrm{v}$ dc power supply, and can provide peripheral microcontroller $5 \mathrm{v}$ output voltage and output current $100 \mathrm{ma}$. When sending signals, I/O module in the modulation signal and power coupling interface circuit, realizes the data transmission. Therefore interface circuit in the signal, it is necessary to filter the processing, and through the power amplifier power amplifier. Also, at the receiving end, interface circuit need to filter and power amplification, signal demodulation finally sent to the modem.

Signal demodulation, such as adaptive amplifier and filtering by the power line carrier communication function the FSK modem chip, following the FSK modem via a serial port and digital signal processor to exchange data, through serial peripheral interface SPI serial interface and the module of communication. The working voltage of electric power carrier module is $6.5 \mathrm{v}$ dc 20 , as a result, the power manager is step-down power management IC, voltage regulation and current regulation is lesser, and built-in frequency compensation circuit and fixed frequency.

When work, in addition, due to the power management module produces strong electromagnetic interference, so power carrier module in PCB design, power management module should as far as possible away from modem module.

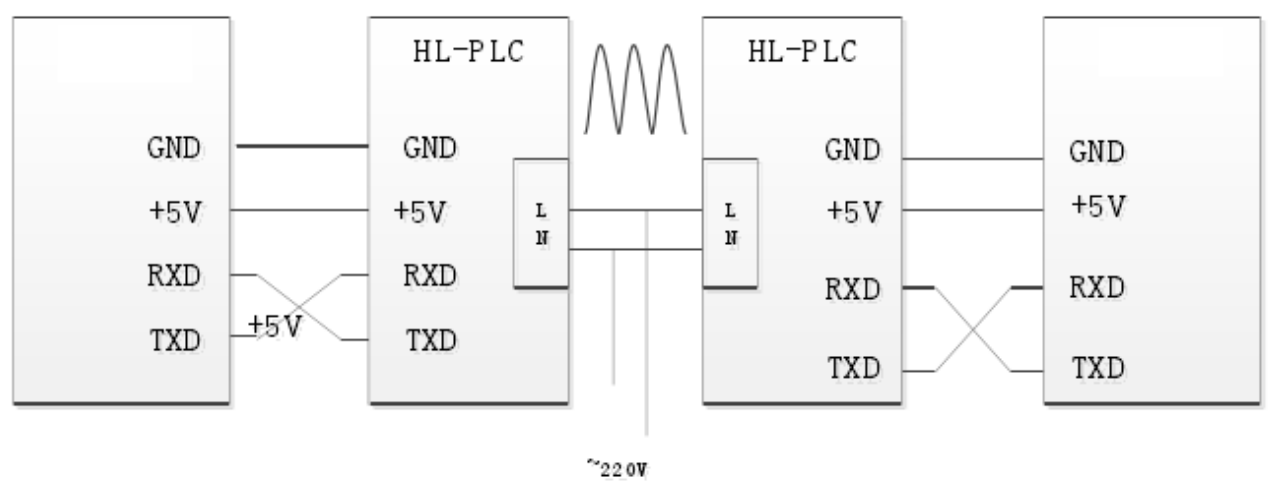

Figure 1. Power carrier communication module design 


\section{MONITOR GATEWAY FUNCTION DESIGN AND IMPLEMENTATION}

The traditional web server is made of high performance computer implementation. The embedded system resources are limited, and no other additional features. So if by single chip microcomputer as a server, need to meet the remote access, monitoring equipment, and other functions. On the client, use the hypertext transfer protocol to communicate with the server.

Before to communicate with the client and server using TCP/IP protocol to establish a socket connection, the browser sends the request through the socket connection, be sent to the server via the Internet, the socket connection port number is 80; Then the server parses the hypertext transfer protocol header files, check promptly returned to the client after legal response, the client and thus established a TCP/IP connection. After the connection is established, the client sends the request can be received by the server. The browser will return results show on the screen. From the above process can be concluded that the web server in the process of communication, the need to complete the receiving, parsing, response, a series of functions, and returned to the client request. The whole process, the application process may take up the same TCP/IP port, need to adopt socket mechanism enables multiple processes sharing a TCP/IP connection.

\section{CONCLUSIONS}

This article through to the independent type fire alarm system based on electric power carrier research and design, mainly the following results:

(1) On dependent type fire smoke alarm system and the overall scheme design and implementation. Gateway through smoke monitoring module, monitoring module and upper desktop module design, solve the independent type of fire alarm system the basic performance requirements.

(2) Data communication module design and implementation. This system to choose the power carrier transmission mode, studies the working process of the electric power carrier communication technology software and hardware, how solved according to the characteristics of the electric power carrier technology, its application field of fire alarm.

(3) The design and implementation of monitor gateway. Monitor gateway is composed of ARM and the router, this system through the domain to dynamic IP parsing and lightweight TCP/IP protocol, and a series of technology, has realized the monitoring of the gateway and the client Socket communication.

(4) The realization of the PC interface. By discussing the client browser and the web server communication process, realize the PC interface environment construction and the client to smoke sensor monitoring.

\section{REFERENCES}

[1] Gong Yunyi, Gu Wenge, Tong Jun . Ignit ion capability of frictions parks and hot surface for fire $r$ isk[J] ., 1999, 8( 1) : 1- 14.)

[2] Katayama M, Yamazato T, Okada H. A Mathematical Model of Noise inNarrowband Power Line Communication Systems[J]. IEEE Journal Selected Areain Communication,2006,24(7):12671276.

[3] Lee1 M K, Newman RE, Latchman H A,et al. Power Line Communication LANs-protocol Description and Performance Results[J]. International Journal of Communication System, 2003, 16: 447-473.

[4] Brendan. Designing the Best Call Center for Your Business Puhlication Date[M]. New York : Harner Row, 2004 : 231-238.

[5] Gajbhiye, P. Mahajan, A. A survey of architecture and node deployment in Wireless Sensor Network. Applications of Digital Information and Web Technologies, 2008,426-430.

[6] Rajakovic N,Nikolic D, Vujasionovic J. Cost benefit for implementation of asystem for remote control and automatic meter reading. In: IEEE Bucharest Power Tech Conference. Bucharest,2009,1-6.

[7] M. Young, The Technical Writer's Handbook. Mill Valley, CA University Science, 19 Sivaneasan B, Gunawan E,So P L. Modeling and performace analysis of automatic meter reading systems using power line communications. In: IEEE Singapore International Conference on Communcation Systems,Singapore, 2008, 14461450

[8] Kwang-il H,Byoung-Jo C,Seok-hoon K. Enhanced selfconfiguration scheme for a robust Zigbee-based home automation IEEE Transactions on Consumer Electronics, 2010,56(2): 583-590:

[9] Khalifa T, Naik K, Nayak A. A Survey of Communication Protocols for Automatic Meter Reading Applications. IEEE Communications Survey \&Tutorials, 2010,PP(99): 1-15

[10] Gomez C, Paradells J. Wireless home automation networks: A survey of architecture and technologies. IEEE Communication Magazine,2010,48(6):92-101 